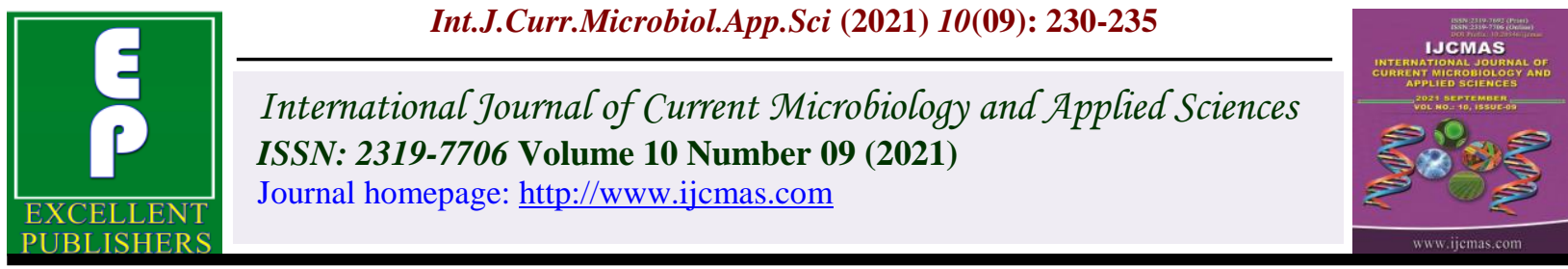

\title{
Determination of Vancomycin and Linezolid Resistance among Staphylococcal Isolates from a Tertiary Care Hospital
}

\author{
R. Shakthi* and D. Venkatesha \\ Department of Microbiology, Adichunchanagiri Institute of Medical Sciences, \\ B.G.Nagara, Mandya, Karnataka, India \\ *Corresponding author
}

\section{A B S T R A C T}

\section{Keywords}

Methicillin resistant Staphylococcus aureus,

Vancomycin, Linezolid,

Minimum

Inhibitory concentration

Article Info

Accepted:

15 August 2021

Available Online:

10 September 2021
Methicillin resistant Staphylococcus aureus (MRSA) has emerged as one of the commonest causes of hospital acquired infections. Vancomycin is the drug of choice for the treatment of MRSA. Increase in Minimum inhibitory concentration (MIC) of Vancomycin has been observed in both Methicillin sensitive and resistant Staphylococcal isolates. In critically ill patients, Vancomycin has become a poor therapeutic antibiotic whereas; Linezolid has emerged as an alternative drug in treating such patients. This study was undertaken to determine the sensitivity pattern among clinical isolates of Staphylococcus aureus (S. aureus) to Vancomycin and Linezolid, in order to formulate a better treatment.100 S. aureuswere isolated from various clinical specimens. Antibiotic sensitivity testing was performed by Kirby Bauer disc diffusion method and MICs of Vancomycin and Linezolid were determined by E-test following CLSI guidelines. Out of $100 \mathrm{~S}$. aureus isolated, 68 were MRSA strains. Among 68 strains of MRSA for which MIC levels of Vancomycin $4 \mu \mathrm{g} / \mathrm{ml}$, $2 \mu \mathrm{g} / \mathrm{ml}$ and $1 \mu \mathrm{g} / \mathrm{ml}$ were 4,27 , and 37 respectively. Similarly, out of total MRSA strains MIC levels of Linezolid $4 \mu \mathrm{g} / \mathrm{ml}, 2 \mu \mathrm{g} / \mathrm{ml}$ and $1 \mu \mathrm{g} / \mathrm{ml}$ were 1,25 , and 42 respectively. All 100 strains showed similar in-vitro efficacy for Vancomycin and Linezolid by Kirby Bauer disc diffusion method, but the number of strains with higher ranges of MICs of Vancomycin were more as compared to those which had higher ranges of MICs for Linezolid. Hence we suggest that Linezolid can be used as an alternative for the treatment of MRSA.

\section{Introduction}

Methicillin resistant Staphylococcus aureus (MRSA) has been emerged as one of the commonest causes of hospital acquired infections globally. The infections caused by MRSA prolong the duration of hospital stay and also increases the financial burden and morbidity. Many studies have reported resistance to all antibiotics available for use against Staphylococcus aureus. Vancomycin has been the most authentic therapeutic agent against MRSA (Rajaduraipandi et al., 2006). The condition has been further worsened by 
the emergence of Vancomycin intermediate sensitive Staphylococcus aureus (VISA) and Vancomycin Resistance Staphylococcus aureus (VRSA) (Loomba P S et al., 2010). Failure of Vancomycin therapy or reduction in its efficacy has been reported among MRSA strains with increased Vancomycin MICs (1-2 $\mu \mathrm{g} / \mathrm{ml}$ or $2-4 \mu \mathrm{g} / \mathrm{ml}$ ) (Tandel et al., 2012). The newly developed Linezolid drug belongs to class of Oxazolidones is probably one of the few choices for the treatment of Vancomycin resistant MRSA (Zurenko et al., 1996).

Hence, this present study was conducted to determine the in-vitro activities of Vancomycin and Linezolid against Staphylococcus aureus and to know the various MICs of both drugs for formulation of better empirical therapy.

\section{Materials and Methods}

This study was conducted in the Department of Microbiology, AH\&RC, B.G. Nagara, between January 2020 to December 2020, with ethical committee approval. 100 Staphylococcus aureus strains were isolated from various clinical specimens like pus, wound swabs, blood, urine, cerebrospinal fluid, catheters, sputum, high vaginal swabs and other body fluids from patients attending OPD and admitted in different wards of AH\&RC. All specimen was processed in the Central laboratory of department of Microbiology. Only non-repetitive clinical isolates were included in this study.

The collected specimens were inoculated on Blood agar, MacConkey's agar plates and they were incubated at $37^{\circ} \mathrm{C}$ for $24-48$ hours. A presumptive identification of organism was done by colony morphology, Gram's staining, Catalase test and Slide coagulase test. A confirmation was done by tube coagulase test, growth on Mannitol salt agar, DNAse test. Antibiotic susceptibility was done by Kirby
Bauer disc diffusion method. Detection of MRSA was done by using Cefoxitin disc (30 $\mu \mathrm{g})$. The Staphylococcus aureus strains with Zone of diameter of $<21 \mathrm{~mm}$ were considered as MRSA and those with $>22 \mathrm{~mm}$ as sensitive. The MICs of Vancomycin and Linezolid were detected by E-test (HiMedia laboratories) as per CLSI guidelines 2019 (Bauer et al., 1966). The zone of inhibition was observed. The zone edge intersecting the graded strip at the minimum concentration of the antibiotic was interpreted as MIC (Fig1 and Fig 2). S. aureus ATCC 29213 was used as control strain. The MICs were interpreted in (Table 1).

\section{Results and Discussion}

A total of 100 Staphylococcus aureus were isolated from 250 clinical specimens from various departments. Majority of the isolates were from pus followed by blood and urine (Table.2).

Out of 100 Staphylococcus aureus isolates, 68 isolates were Methicillin Resistant Staphylococcus aureus and the remaining isolates were Methicillin sensitive. All 100 isolates were sensitive to Vancomycin $(30 \mu \mathrm{g})$ and Linezolid $(30 \mu \mathrm{g})$ by Kirby Bauer disc diffusion method. No Vancomycin or Linezolid Resistant Staphylococcus aureus strains were detected by determination of MICs using E- test. MICs between 0.5-2 $\mu \mathrm{g} / \mathrm{ml}$ for Vancomycin and $1-4 \mu \mathrm{g} / \mathrm{ml}$ for Linezolid were obtained for all 100 strains isolated from clinical specimens by E-test. Table 3 and Table 4 shows number of strains with various concentrations of MICs for Vancomycin and Linezolid. Fig 3 shows comparison of MIC of Vancomycin and Linezolid.

Out of 68 MRSA strains for which MIC levels of Vancomycin $4 \mu \mathrm{g} / \mathrm{ml}, 2 \mu \mathrm{g} / \mathrm{ml}$ and $1 \mu \mathrm{g} / \mathrm{ml}$ were 4, 27 and 37 respectively. Similarly, out of total MRSA strains MIC levels of Linezolid 
$4 \mu \mathrm{g} / \mathrm{ml}, 2 \mu \mathrm{g} / \mathrm{ml}$ and $1 \mu \mathrm{g} / \mathrm{ml}$ were 1,25 , and 42 respectively (Figure 4 ).

In this study, Staphylococcus aureus was the commonest pathogen isolated from various clinical specimens especially from pus, wound swabs collected from patients with localized pyogenic and surgical wound infections admitted in surgery and orthopedic wards, which is in correlation with other studies (Gupta et al., 2003).

All 100 Staphylococcus aureus isolates were sensitive to both Vancomycin and Linezolid by Kirby Bauer disc diffusion method, but Vancomycin showed comparatively higher MICs than Linezolid by E-test. All 68 MRSA strains were also more susceptible to Linezolid. These findings are similar with the studies of Sachin et al., (2015), Srinivasan et al., (2006) and Kaleem et al., (2011). These studies also emphasize that Linezolid is a good therapeutic option for treatment of MRSA.

In this study, 4 Staphylococcus aureus strains showed MIC of $4 \mu \mathrm{g} / \mathrm{ml}$ for Vancomycin, these strains were isolated from the patients admitted in Orthopedic (3) and Surgery wards (1). Long duration of stay in hospital and prolonged antibiotic therapy for these patients may be the cause of higher MICs and multidrug resistance. The chronic exposure for antibiotics may lead to selective antibiotic resistant strains, these findings are in correlation with other studies (Rajaduraipandi, et al., 2006, Sachin, et al., 2015). In the present study, all 100 Staphylococcus aureus were sensitive to Linezolid by Kirby Bauer disc diffusion method, which is similar to other studies (Sachin K et al., 2015)

Table.1

\begin{tabular}{|c|c|c|}
\hline \multirow{2}{*}{ MIC } & \multicolumn{2}{|c|}{ MICs of Antibiotics in $\boldsymbol{\mu g} / \mathbf{m l}$} \\
\cline { 2 - 3 } interpretation & Vancomycin & Linezolid \\
\hline Sensitive & $\leq 2$ & 4 \\
\hline Intermediate & $4-8$ & - \\
\hline Resistance & 16 & - \\
\hline
\end{tabular}

Table.2 Distribution of Staphylococcal aureus isolated from the various clinical specimens

\begin{tabular}{|c|c|c|}
\hline Type of specimen & Number of specimen & S.aureus (\%) \\
\hline Pus & 126 & 52 \\
\hline Blood & 50 & 22 \\
\hline Urine & 55 & 18 \\
\hline Sputum & 6 & 3 \\
\hline High Vaginal swabs & 6 & 3 \\
\hline CSF & 2 & - \\
\hline Catheters and Other body \\
fluids
\end{tabular}


Table.3 MICs of Staphylococcus aureus for Vancomycin using E-test

\begin{tabular}{|c|c|}
\hline Minimum Inhibitory Concentration & Vancomycin \\
\hline No of Strains with MIC $\leq \mathbf{4} \boldsymbol{\mu} \mathbf{g} / \mathbf{m l}$ & 4 \\
\hline No of Strains with $\mathrm{MIC} \leq \mathbf{2} \boldsymbol{\mu \mathbf { g } / \mathbf { m l }}$ & 30 \\
\hline No of Strains with $\mathrm{MIC} \leq \mathbf{1} \boldsymbol{\mu} \mathbf{g} / \mathbf{m l}$ & 66 \\
\hline
\end{tabular}

Table.4 MICs of Staphylococcus aureus for Linezolid using E-test

\begin{tabular}{|c|c|}
\hline Minimum Inhibitory Concentration & Linezolid \\
\hline No of Strains with $\mathrm{MIC} \leq \mathbf{4} \boldsymbol{\mu} \mathbf{g} / \mathbf{m l}$ & 1 \\
\hline No of Strains with $\mathrm{MIC} \leq \mathbf{2} \boldsymbol{\mu} \mathbf{g} / \mathbf{m l}$ & 54 \\
\hline No of Strains with $\mathrm{MIC} \leq \mathbf{1} \boldsymbol{\mu} \mathbf{g} / \mathbf{m l}$ & 45 \\
\hline
\end{tabular}

Fig.1 Vancomycin E-strip test showing sensitive MIC

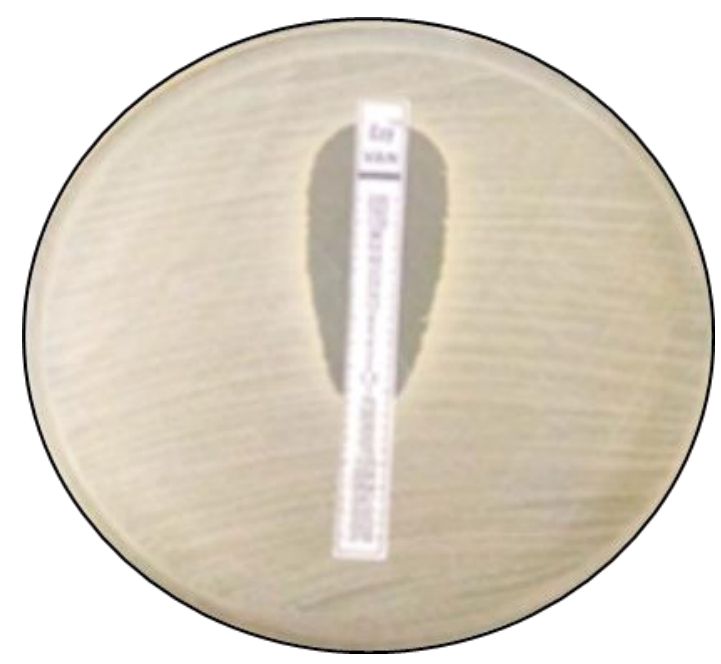

Fig.2 Linezolid E-strip test showing sensitive MIC

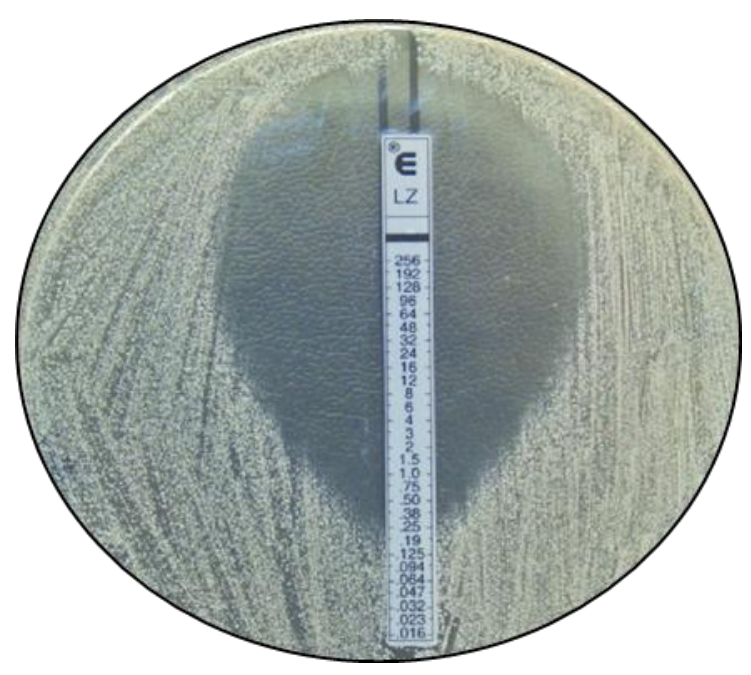


Fig.3 Comparison of MIC of Vancomycin and Linezolid

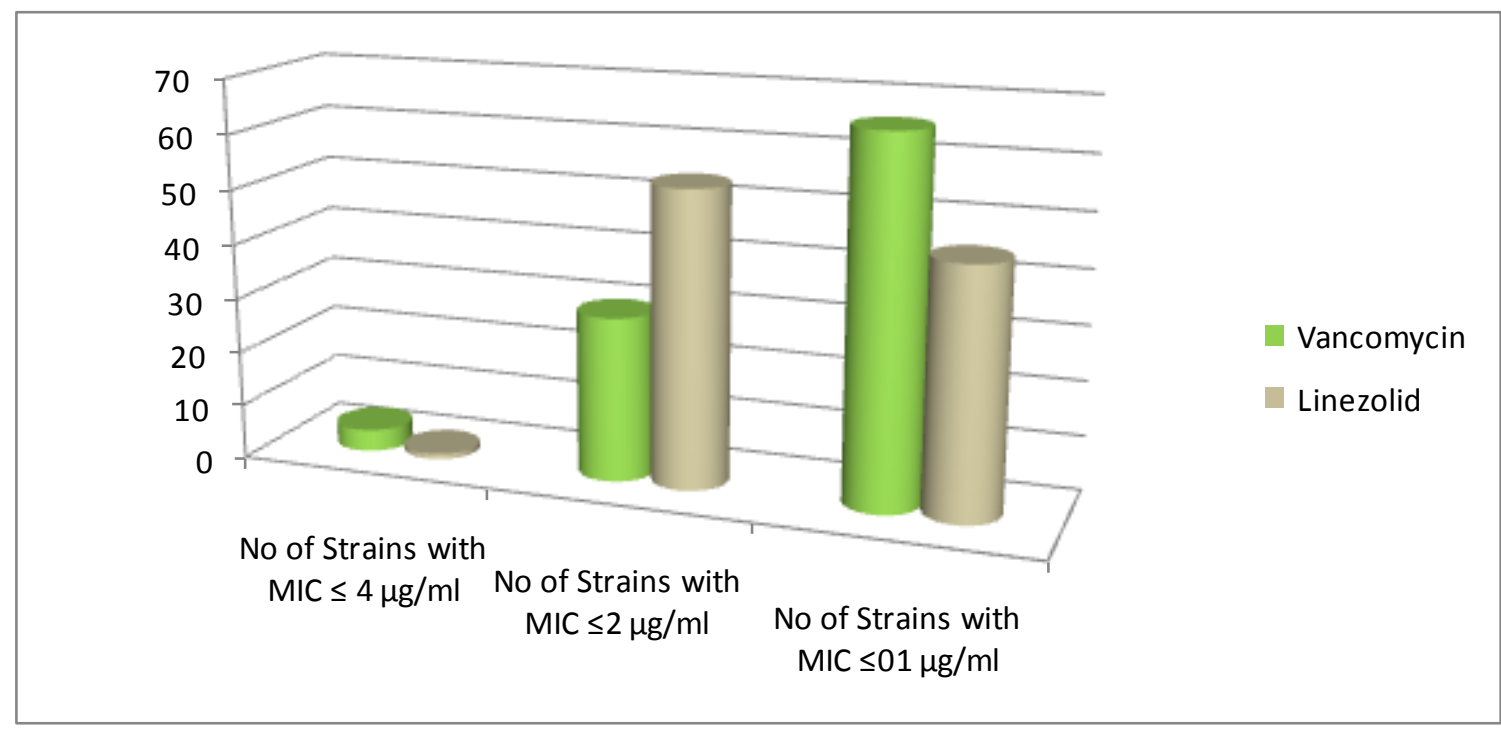

Fig.4 Comparison of MIC of Vancomycin and Linezolid for MRSA strains

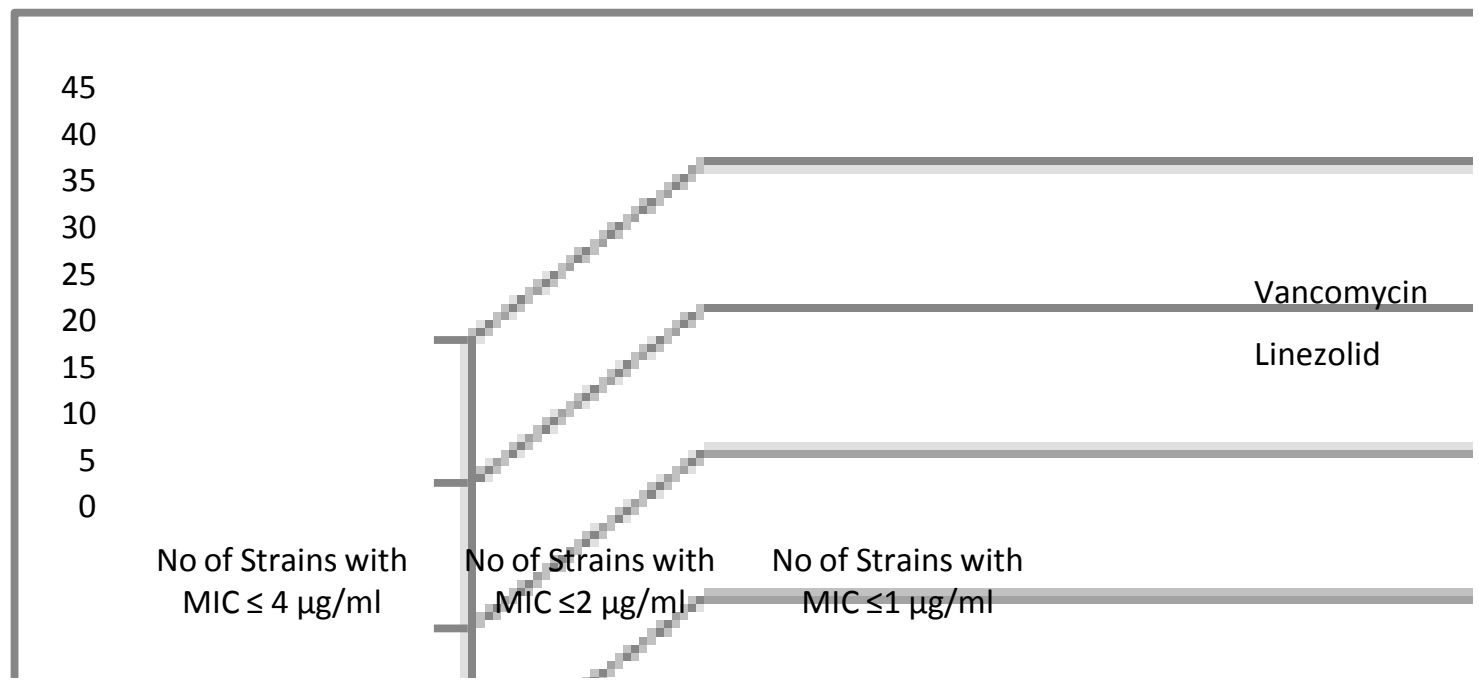

Other studies have reported that continuous monitoring (Shinabarger et al., Staphylococcal strains with upper level MICs 1997). In one of the study for MRSA of Vancomycin will lead to more morbidity infections Linezolid showed desired, and mortality among patients compared to improved outcome compared Vancomycin those with lower MICs (Price et al., 2009).

Vancomycin requires intravenous administration, monitoring of levels and rarely patients may experience some side effects. However, Linezolid is available in both intravenous and oral form. Linezolid can be rapidly and completely absorbed following oral administration; it doesn't require
(Howden Benjamin et al., 2004).

According to this study, both Vancomycin and Linezolid have similar in-vitro efficacies for MRSA infections. Oral dosing of Linezolid leads to earlier discharge of patients and cost effective. Along with in-vitro efficacies Linezolid also have good in-vivo efficacy, which makes it an important therapeutic 
alternative to Vancomycin in treatment of MRSA infections.

The indiscriminate use of antibiotics leads to emergence of resistance. As only limited drugs are available for the treatment of MRSA, a good antibiotic policy should be implemented in all tertiary care hospitals and a strict antibiotic regimen should be applied by clinicians. So we suggest that Linezolid is a good alternative therapeutic drug for the treatment of multidrug resistant Staphylococcus aureus.

\section{References}

Bauer, Kirby, Sherris and Turck, 1966, Am. J. Clin. Path., 45: 493.

Gupta N, Aparna, Saini S, Kumar B Arora DR. In vitro activity of Linezolid in Staphylococcus aureus. IJMM. (2003); 21(4):289-90.

Howden P Benjamin, Ward B Peter, Charles GP Patrick, Korman M Tony, Fuller Andrew, Cros du Philipp, et al., Treatment Outcomes for Serious Infections Caused by Methicillin-Resistant Staphylococcus aureus with Reduced Vancomycin Susceptibility. Oxford Journals, Medicine, Clinical Infectious Diseases. 2004;38(4): 521-8.

Fatima, K, Usman Javaid, Khalid Ali, Hassan Afreenish, Omair Maria, et al., Comparison of in vitro efficacy of linezolid and vancomycin by determining their minimum inhibitory concentrations against methicillin resistant Staphylococcus aureus (MRSA). April, 2011.

Tandel, K, A K Praharaj, Kumar S, et al., Differences in vancomycin MIC among MRSA isolates by agar dilution and $\mathrm{E}$ test method. IJMM. 2012; 304(4):453-5.
Loomba P S, TanejaJuhi, Mishra Bibhabati, et al., Methicillin and vancomycin resistant S. aureus in hospitalized patients. Journal of Global Infectious Diseases.2010;2(3):27583.

Performance standards of Antimicrobial Susceptibility Testing; Twenty Ninth Informational Supplement.M100-S29, Vol. 39, No.1, Jan 2019.

Price J, Atkinson S, Llewelyn M, Paul J, et al., Paradoxical relationship between the clinical outcome of $\mathrm{S}$. aureus bacteremia and the minimum inhibitory concentration of vancomycin. Clin Infect Dis. 2009; 48:997-8

Rajaduraipandi K, Mani K R, Panneerselvam K, Mani M, Bhaskar M, Manikandam P. Prevalence and antimicrobial susceptibility pattern of Methicillin Resistant Staphylococcus aureus: A multicenter study. Indian JMed Microbiol.2006;24:34-8.

Sachin K, Deepika V, Me Siddique., Comparison of In-vitro Activities of Linezolid and Vancomycin against Staphylococcus aureus Isolated from A Tertiary Care Hospital'.JCDR 2015;8(5):12-5.

Shinabarger D L, Marotti K R, Murray R W, Lin A H, Melchior E P, Swaney S M, et al., Mechanism of action of the oxazolidiones: effect of linezolid and eperzolid on translation reactions. Antimicrobial agent chemotherapy. 1997; 41:2132-6.

Srinivasan S, Sheela D, Mathew R, Bazrocy J, Kanungo R. Risk factors and associated problems in the management of infections with methicillin resistant $S$. aureus. IJMM. 2006; 24(3):182-5.

Zurenko G E, Yagi B H, Schaadt R D, Allison J W, Kilburn J O, Glickman S E, et al., In vitro activities of U-100592 and U-100766, novel oxazolidinone antibacterial agents. Antimicrob Agents Chemother. 1996;40:839-45.

\section{How to cite this article:}

Shakthi, R. and Venkatesha, D. 2021. Determination of Vancomycin and Linezolid Resistance Among Staphylococcal Isolates from A Tertiary Care Hospital. Int.J.Curr.Microbiol.App.Sci. 10(09): 230-235. doi: https://doi.org/10.20546/ijcmas.2021.1009.026 